\title{
Presence of voids after three obturation techniques in band-shaped isthmuses: a micro- computed tomography study
}

Pengfei Zhang ${ }^{1,2}$, Keyong Yuan ${ }^{1,2}$, Qiaoqiao Jin ${ }^{1,2}$, Fen Zhao ${ }^{1,2}$ and Zhengwei Huang ${ }^{1,2^{*}}$

\begin{abstract}
Background: The objective of the present micro-computed tomography (micro-CT) study was to assess the presence of voids in band-shaped isthmuses obturated using three different filling techniques.

Methods: Twenty-four artificial molar teeth with a band-shaped isthmus were allocated to three groups $(n=8)$ for obturation, according to the filling technique: single-cone (SC), continuous wave of condensation (CWC) or lateral condensation (LC). Obturation was performed with gutta-percha (GP) cones and iRoot SP (Innovative Bioceramix, Vancouver, Canada). Post-filling micro-CT scanning was performed. The percentage of filling materials and void volumes were calculated in the isthmus areas and data were analyzed using one-way ANOVA and Tukey tests.
\end{abstract}

Results: The mean percentage of void volumes and corresponding filling percentages in the isthmus areas after obturation in the SC groups was $22.98 \% \pm 1.19 \%, 77.02 \% \pm 1.19 \%$; in the CWC groups $10.46 \% \pm 2.28 \%, 89.54 \% \pm$ $2.28 \%$; and in the LC groups was $13.14 \% \pm 1.85 \%, 86.86 \% \pm 1.85 \%$, respectively.

Conclusions: In band-shaped isthmus area, the obturation quality of CWC was superior to SC and LC techniques.

Keywords: Isthmus, micro-CT, Obturation techniques, Voids

\section{Background}

The goal of endodontic treatment is the removal of harmful microorganisms from the root canal to prevent or treat apical periodontitis [1]. Apart from the proper cleaning and shaping of the root canal, adequate obturation of the root canal system is also required after biomechanical procedures, to ensure the long-term success of endodontic treatment [2]. However, it is difficult to achieve this goal because of anatomical complexities of the root canal system and limitations in current preparation and filling techniques $[3,4]$. Approximately $60 \%$ of endodontic failures are due to insufficient obturation of

\footnotetext{
*Correspondence: huangzhengwei@shsmu.edu.cn

${ }^{1}$ Department of Endodontics, Shanghai Ninth People's Hospital, Shanghai

Jiao Tong University School of Medicine, College of Stomatology,

Shanghai Jiao Tong University, Shanghai 200011, China

Full list of author information is available at the end of the article
}

the root canal system [3]. Inadequate obturation has been reported to result in voids within the canal filling and in the interface between filling and dentin, which permits bacterial movement in the coronal-apical direction or vice versa, potentially leading to reinfection or persistent apical periodontitis $[5,6]$. Therefore, the association of high quality root canal obturation with treatment success has been emphasized [3, 5-8].

The variations and complexity of root canal anatomy such as isthmuses bring a key challenge in endodontic therapy of posterior teeth [4,9]. An isthmus (also termed as a lateral interconnection, corridor, or transverse anastomosis) is a narrow, ribbon-shaped communication between two root canals $[4,9,10]$. A band-shaped isthmus is defined as a band-shaped corridor between root canals starting from the level where two canals merge (isthmus roof) and extending to the 
level where two root canals redivide (isthmus floor) [10]. The highest frequencies of isthmuses in human permanent teeth are found in the lower first molars, and their reported prevalence ranges from 50 to $80 \%$ at the apical $5 \mathrm{~mm}$ level, evaluated using surgery and micro-CT methods [9-11].

The isthmuses remain one of the most difficult clinical challenges during root canal treatment, owing to accumulation of hard and/or soft tissue debris which can contain biofilms and intracanal microorganisms, after canal preparation within these areas $[12,13]$, leading to treatment failure. Therefore, the choice of root canal filling materials and obturation technique is very important in order to achieve a high quality seal in isthmus areas.

Most root canal filling techniques employ a core material, which is most commonly gutta-percha, and a sealer used to seal the space between gutta-percha (GP) and the canal wall. The traditional technique of lateral condensation (LC), is the most classic method of clinical root canal obturation and has been used for many years in numerous studies. Meanwhile, thermoplastic sealing techniques, such as continuous wave of condensation (CWC), have been reported to be advantageous for the management of irregular root canals [14, 15]. In addition, the single cone (SC) technique has become increasingly popular due to its easier implementation, better adaptation in three-dimensional preparation, low cost and short operation time [7, 14-17]when applied with a bioceramic sealer. Although a few studies [14-18] have used human molar teeth to evaluate the quality of obturation with different filling techniques, the variation was large because of complex canal anatomy shapes among different samples. For this reason, when measuring the percentage of voids in root canal fillings, three-dimensional (3D)printed artificial teeth can provide more reliable information. However, there are still few reports involving a band-shaped isthmus [18].

Micro-computed tomography (micro-CT) scanning is an emerging technology with multiple applications in endodontic research $[7,9,10,14-18]$. In terms of root canal filling, compared to conventional imaging techniques, such as scanning electron microscopy or confocal microscopy, micro-CT imaging is a nondestructive and visual method to calculate the volume of filling material without damaging the specimen, providing high-resolution 3D assessment of the quality of filling [15-21].

This study aimed to evaluate and compare the canal filling quality when performing different techniques on 3D-printed artificial teeth with band-shaped isthmuses by calculating the percentage of voids which was analyzed using micro-CT. The null hypothesis was that the percentage of voids in isthmus areas would not be influenced by filling technique.

\section{Methods}

\section{Preparing 3D-printed artificial molar teeth}

The teeth were customized samples reproducing the shape of the human mandibular first molar, which possessed Vertucci classification [4] type VI canals in the mesial root. Mesial-buccal (MB) and mesial-lingual (ML) canals with a $1 \mathrm{~mm}$ diameter and $4 \mathrm{~mm}$ distance at the orifice, were relatively straight and were joined together $5 \mathrm{~mm}$ from the apex and continued to the apex eventually. Thus, there was a root canal anatomy of "bandshape isthmuses" [10, 18] present in the mesial root of these teeth, with a roof and floor which exhibited a long, oval cross-sectional shape. The isthmus roof was at apex $5 \mathrm{~mm}$,and its floor was at apex $1 \mathrm{~mm}$. The buccallingual width of it was $1.5 \mathrm{~mm}$,and the mesial-distal width of it was $0.2 \mathrm{~mm}$. This was the focus of our research, so the follow-up processing only involved the mesial root, especially the apical $5 \mathrm{~mm}$ region. The image data with DICOM style acquired from the micro-CT (Skyscan 1172; Bruker-Micro-CT, Kontich, Belgium) scans was exported to Mimics ${ }^{\circledR}$ software (version 21.0; Materialise Corporation, Leuven, Belgium) to conduct 3D reconstruction and generate 3D printing model. Then 3D printing model data was exported to 3D printing software, through which the 3D-printed artificial molar teeth were produced at a printing precision of $0.016 \mathrm{~mm}$ on a 3D Systems Projet MJP3510 HD printer (3D Systems, Inc., Rock Hill, USA) with multi-jet printing technology (Multi-Jet Printing System, MJP).The crown of the teeth was generated by VeroWhite Plus RGD835 and the root was made of VeroClear RGD810 (Typrius,Inc.Beijing, China),which both had similar elastic modulus with respective part of human teeth and had a melting point at 375 and $350^{\circ} \mathrm{C}$ respectively.

\section{Samples selection and root canal preparation}

Based on the data of previous studies, a power beta of 0.95 by using G*Power 3.1 (Heinrich Heine University, Düsseldorf, Germany) was set, indicated a minimum expected sample size for each group should be six requiring to observe the same effect $(3.48)[15,19]$. Then, eight 3D-printed artificial molar teeth for each obturation group were selected for this study to assess the void percentage in isthmus areas. The crowns of the artificial teeth were cut horizontally from the cervix for the convenience of operation. Then working length (WL) was determined using an ISO size \#10 K-file (Dentsply, Switzerland) inserted into the canals until the tip was just visible beyond the apex with a dental operating microscope (Zeiss, Gottingen, Germany) at a magnification of $8 \times$ and was measured by deducting $1 \mathrm{~mm}$ from this length. Then, both mesial canals were instrumented, applying a crown-down technique using ProTaper Gold 
$\mathrm{Ni}-\mathrm{Ti}$ system (Dentsply, Switzerland) coupled with the Dentsply X-Smart motor (Dentsply, Switzerland) in the sequence recommended by the manufacturer, reaching to F3. A new file was used on each specimen. Every time the instrument was withdrawn, the canals were irrigated with physiological saline delivered by a 30 -gauge needle (Monoject, Covidien, Dublin, Ireland). After preparation, the canals were dried with paper points (Dentsply, Switzerland).

After preparation, to evaluate the consistency of canal shapes and homogeneity of isthmus figures, three teeth were randomly selected for micro-CT scanning, and the obtained images were overlapped [21].

\section{Root canal filling}

The overall 24 specimens were randomly allocated into three groups containing eight teeth each group. These specimens were filled using the single-cone (SC), lateral compaction (LC), or continuous wave of condensation (CWC) technique. The iRoot SP (Innovative Bioceramix, Vancouver, Canada) was used as the root canal sealer according to the manufacturers' instructions in all three obturation procedures.

Group 1 was obturated using the SC technique [1418]. The canals were first fully injected with iRoot SP via a 24-gauge needle tip provided by the manufacturer. The tip was slowly pulled toward the orifice from the point of engagement in the canal. Subsequently, a lentulo spiral was placed in the canal, to allow a thin sealer to cover the canal wall evenly. Then, a single F3 ProTaper GP cone (Dentsply, Switzerland) modified to the working length with sealer was slowly inserted into the canals. A heat plugger (B\&L Biotech, Ansan, Republic of Korea)was used to cut excess filling material at the orifice level, and the cone was vertically condensed using a cold plugger.

Group 2 was obturated using the CWC technique [15, $18,19]$. The root canals were coated with a thin layer of sealer using a paper point, then the F3 GP cone (Dentsply, Switzerland) was fitted as a master cone with sealer placed to the WL. A B\&L-alpha II tip (B\&L Biotech, Ansan, Republic of Korea) setted at $180{ }^{\circ} \mathrm{C}$ was inserted into the canal to cut the master cone, retaining only the apical GP $(5 \mathrm{~mm})$. Next, the coronal portion of the canal was backfilled with B\&L-Beta (B\&L Biotech, Ansan, Republic of Korea) setted at $200{ }^{\circ} \mathrm{C}$ to the canal orifice, and vertically condensed with suitable hand pluggers.

Group 3 was obturated using the LC technique [7, 14]. The appropriate 35/0.02 tapered GP cone (Dentsply, Switzerland) modified to the WL as a master cone, was coated with a thin layer of sealer and slowly inserted into the root canal. Then the lateral compaction procedure was performed with a size 25 nickel-titanium finger spreader (Dentsply Tulsa, Switzerland) and size 20/0.02 taper accessory GP cones (Dentsply, Switzerland). After that, the coronal excess was seared off at the canal entrance and the remaining filling material was vertically condensed using a cold plugger.

A single professional endodontist(Z.W.H) with more than 15 years' clinical experience performed all root canal preparation and filling procedures to ensure consistency. After obturation, specimens were stored at $37^{\circ} \mathrm{C}$ with $100 \%$ humidity for 2 weeks to confirm that the filling materials had set completely.

\section{Computerized microtomography and microscopy observation}

Because the band-shaped isthmuses were in the apical $5 \mathrm{~mm}$ region of the specimen, first, three artificial tooth specimens after preparation were randomly selected to assess the prepared canal space shapes and discrepancies of isthmus figures among specimens by using the overlapped micro-CT images.

The obturated mesial roots of 3D-printed artificial teeth were scanned using high-resolution micro-CT as previously reported [21], operated at $100 \mathrm{kV}$ and $278 \mathrm{~mA}$ using a $0.5 \mathrm{~mm}$-thick aluminum filter and $40 \%$ beamhardening reduction, which had rotational steps of $0.5^{\circ}$ and a cross-sectional pixel size of $18.30 \mathrm{~mm}$. Scanning was conducted at $360^{\circ}$ rotations around the vertical axis with a camera exposure time of $500 \mathrm{~ms}$. The detector was calibrated before each scan, to minimize ring artifacts. From these scans, cross-sectional images were acquired using NRecon software (version 1.6.9.18; BrukerMicro-CT).Then, the image data with DICOM style was exported to Mimics ${ }^{\circledR}$ software to conduct 3D reconstruction. The range of measurements was $5 \mathrm{~mm}$ from the mesial root apex. The grayscale range was determined in a density histogram by using a global threshold method. With regard to filling material segmentation (GP and sealer), threshold values ranged from 40 to $255 \mathrm{Vm}$, and a grayscale range between 0 and $40 \mathrm{Vm}$ was designated as a void. Finally, 3D models of isthmus region at $5 \mathrm{~mm}$ from the mesial root apex were created for volumetric calculations using Mimics ${ }^{\circledR}$ software. Select the filling material pattern in the 3D model, and then click "Threshold Region Grow" to acquire filling material volume (Filling Vol). Then, The volume of voids was calculated by subtracting the Filling Vol from the post-preparation isthmus volume (Isthmus Vol). The percentage of voids (Voids Vol $\%)$ of the isthmus was calculated as follows:

$$
\text { Voids Vol \% }=(\text { Isthmus Vol }- \text { Filling Vol }) \times 100 / \text { Isthmus Vol }
$$


Because the point of this study focused on the bandshape isthmus present in the apical $5 \mathrm{~mm}$ region of the mesial root, in each specimen, the volume of the filling and voids in the isthmus were measured, and the percentage of voids and filling were calculated.

After micro-CT analysis, the roots were horizontally crossed-sectioned precisely at the middle of the isthmus, which was $3 \mathrm{~mm}$ away from the apex, using a low speed diamond wheel (Isomet, Buelhler Ltd, Dusseldorf, Germany) under water cooling. Each cross-sectioned surface was observed under a microscope at $16 \times$ magnification.

\section{Statistical analysis}

The Shapiro-Wilk test was used to verify whether the data were normally distributed. The data showed normal distribution and homogeneous variance as well as the mean percentage. One-way ANOVA was used to assess differences in the void percentages among the three groups. GraphPad Prism 8 software (GraphPad Software Inc, La
Jolla, CA, USA) was used for all analyses. A $P$ value of $<0.05$ was considered to indicate statistical significance.

\section{Results}

After root canal preparation, artificial tooth specimens $(n=3)$ were scanned in order to evaluate the shape of the prepared canal space and discrepancies among specimens (Fig. 1). The differences between specimens was negligible, with similar volumes of the prepared space of the "band-shape isthmus" in the three samples $\left(1.01982 \pm 0.06 \mathrm{~mm}^{3}\right)$.

Analysis of micro-CT 3D reconstructions revealed the mean volume of voids in the focused area after obturation in the SC, CWC, and LC groups was 0.23436 , 0.10668 , and $0.13394 \mathrm{~mm}^{3}$ respectively, with percentage values of $22.98 \% \pm 1.19 \%, 10.46 \% \pm 2.28 \%$, and $13.14 \%$ $\pm 1.85 \%$ (Fig. 2). Further, the filling percentages of the three groups were $77.02 \%, 89.54$ and $86.9 \%$ correspondingly. The SC group was found to have a significantly greater volume percentage of voids in isthmus obturation $(P<0.01)$ than the other two techniques. In addition, the
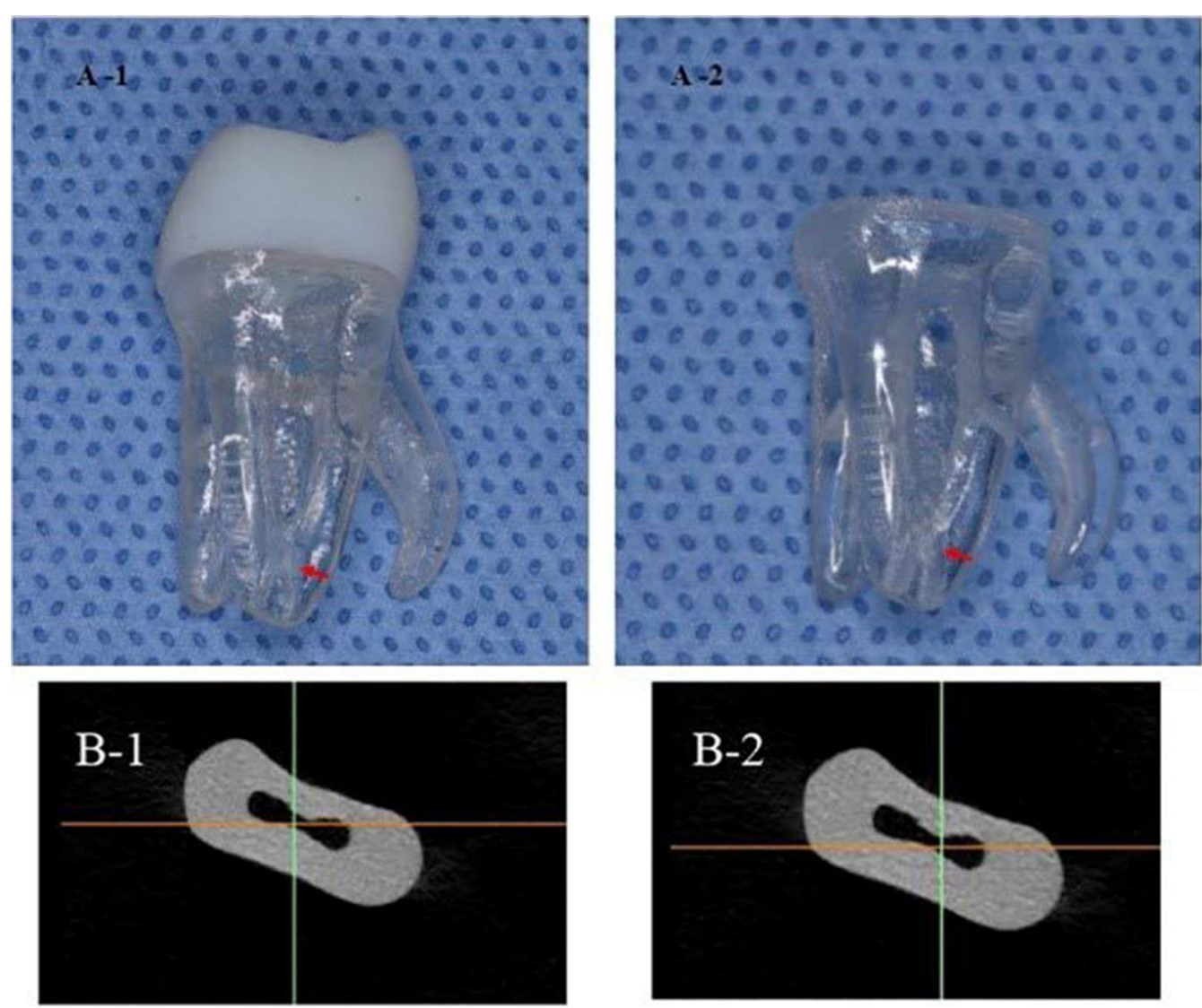

Fig. 1 A 3D printed artificial teeth. Red arrows indicate isthmus.(1) Integral artificial molar teeth. (2) artificial molar teeth without crowns used in this experiment; B Superimposition images of the canals after preparation. From axis view, the differences between specimens could be negligible. (1) The overlapping image of sample 1 and 2. (2) The overlapping image of sample 1 and 3 


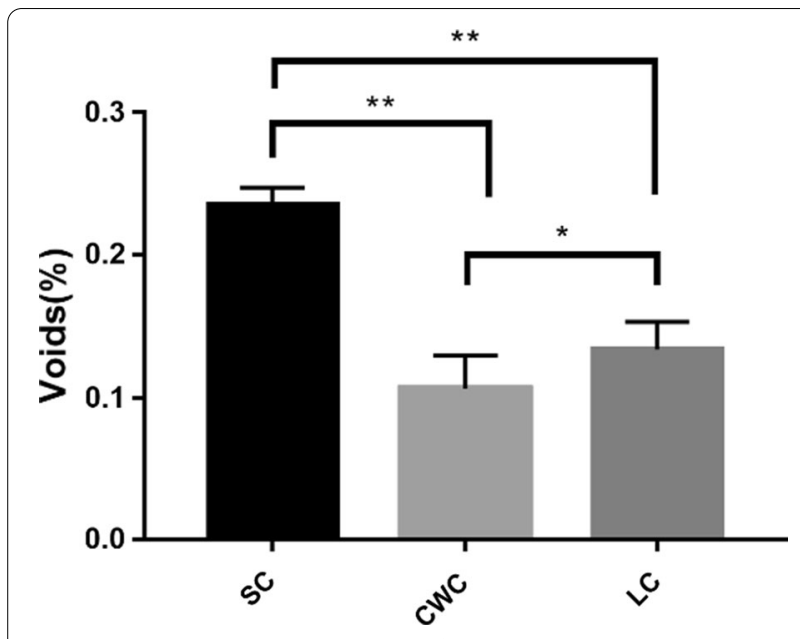

Fig. 2 Volume percentage of voids after obturation in the isthmus areas in three groups. ${ }^{\text {*' }}$ represents statistically significant differences between the groups $(p<0.05)$, while ${ }^{* *^{*}}$ represents $p<0.01$. SC,single-cone technique; LC, lateral condensation technique; CWC,continuous wave of condensation technique

filled volume ratio in LC isthmus areas was significantly lower than that in the CWC group $(P<0.05)$.

Two-dimensional axial cross-sections of representative specimens showed the voids in the root canal filling mass (Fig. 3). Voids were present both inside fillings as well as between the filling materials and root canal walls. Operating microscopic observation demonstrated that the CWC and LC groups were both well-packed, without voids in the middle of isthmuses. In the SC group, the void formation in the middle of the isthmus was clearly visible (Fig. 4).

\section{Discussion}

Successful root canal therapy depends mainly on controlling pulp space infection, which can be achieved by good cleaning and shaping followed by three-dimensional filling of the entire root canal system $[1-3,6]$. However, these steps are frequently challenged by the characteristics of root canal anatomy such as isthmuses. It is reported that the apical $5 \mathrm{~mm}$ region of the mandibular first molar mesial root canals tends to have the highest prevalence of isthmuses, which appear long oval in crosssectional shape with the largest diameter in the buccolingual direction at 1 to $5 \mathrm{~mm}$, which is not evident on periapical radiographs [4, 9-11]. Several studies have pointed out that increased risk of failure of endodontic treatment is associated with the presence of isthmuscontaining canals, owing to the fact that this irregular area may harbor a significant number of microorganisms that might lead to treatment failure [22-24]. In the
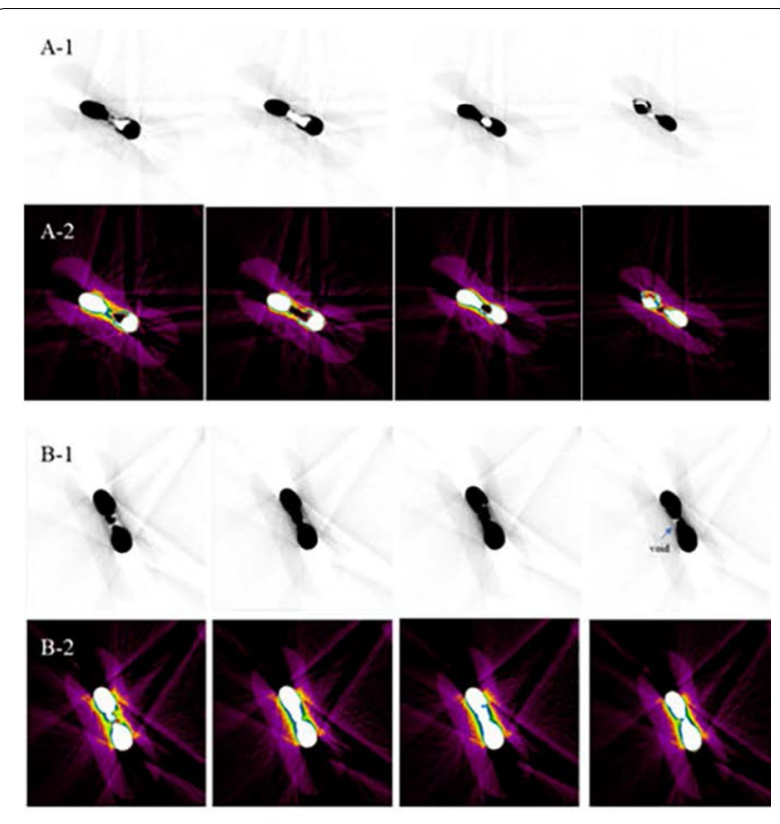

C-1
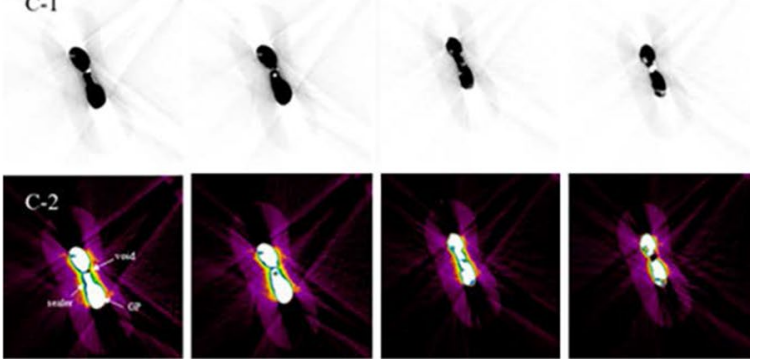

Fig. 3 Representative micro-CT images of three specimens from three groups: A SC, B CWC and $\mathbf{C} L C$ technique groups. The lower row in (A), (B) and (C) is color-coded to distinguish the filling materials. The sealer, void, and gutta-percha (GP) are marked with arrows. (1) and (2) also present representative and consecutive micro-CT cross-sections of one specimen

present study, the null hypothesis was rejected since significant differences were found among the obturation techniques.

Consequently, the majority of previous similar studies have used extracted natural molar teeth to reproduce the clinical situation of isthmus filling $[9,15,18]$. However, the wide variability of isthmuses makes standardization between groups difficult $[10,18]$. Instead, custom-made 3D-printed artificial molar teeth, which had a fused mesial root with canals and a band-shaped isthmus in the apical $5 \mathrm{~mm}$, were used in the present study.To the best of our knowledge, this is the first report to use these artificial teeth in a band-shaped isthmus study.The artificial teeth selected have the same type, volume, length and dimensions, guaranteeing the homogeneity of experimental groups, which makes it possible to obtain more 


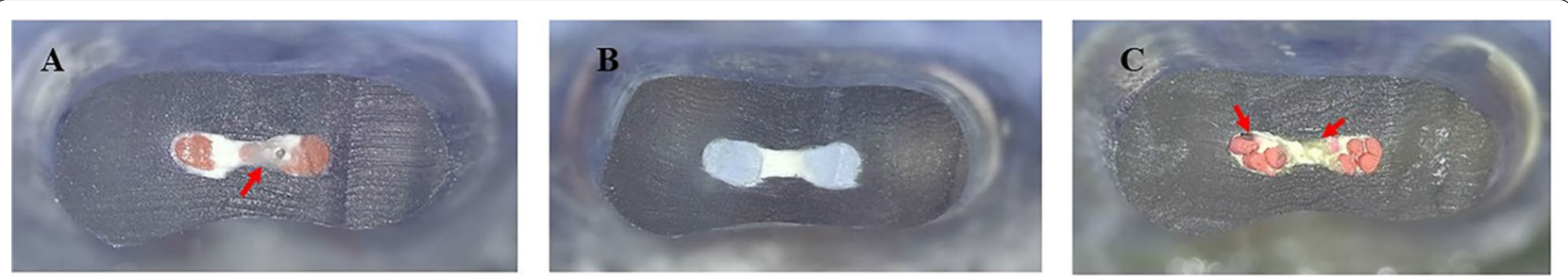

Fig. 4 Operating microscope images (16x) of filling materials in the middle of isthmus in three groups: (A) SC group; (B) CWC group;(C) LC group. The arrow indicates a visible void

reliable results in terms of the void percentages in the isthmus regions after obturation.

Although uniform samples were used in the experiment, the prepared canal shapes may have an influence on the obturation results. Therefore, in order to assess whether the shape of the isthmuses in the mesial root after preparation were consistent, three prepared artificial teeth were randomly selected to obtain micro-CT images as previously reported [21, 25]. The acquired images of the three samples were superimposed, and the results showed no obvious displacement in the location of the isthmus' roof and floor of specimens. Thus the tooth-to-tooth variation can be considered negligible. This may probably because the original canal and isthmus space were quite wide, thus, the conventional root canal preparation had a limited impact on the root canal space of 3D-printed artificial teeth. Consequently, we could maintain a consistent isthmus space volume in all specimens.

Voids percentage has been used as a method to evaluate the quality of root canal filling [14-20], because bacteria and their byproducts may remain at a site in non-obturated areas and affect endodontic treatment outcome. Therefore, adequate sealing of the root canal system is required to prevent the spread of microorganisms and toxins [26].

Previous studies have reported slightly different criteria for dividing the root area to calculate the void percentage. Iglecias et al. [15] divided the root into three areas: apical, middle, and cervical, to assess the void percentage. Kim et al. [25] divided the apical region into 1-5 mm and 5-9 $\mathrm{mm}$ areas for measurement, because they considered this measurement to be more clinically relevant in root canal treatment success than measurements of the full canal length. Somma et al. [26] analyzed the entire root canal system as a whole without division into thirds. In the present study, a similar approach [26]was preferred and a statistically significant difference was detected between different obturation techniques. It is worth noting that according to the findings of the present study, all three obturation techniques were subject to failure, i.e., none were able to completely fill the isthmus region, which is similar to the findings of most previous studies $[7,14-20]$.

In this study, there were significantly more voids in the samples treated by the SC technique than with the CWC and LC methods in filling of isthmuses, with a percentage of $22.98 \%$. This result was consistent with that of several earlier studies [7, 17]. This may be because, unlike the other two techniques, the SC technique lacks vertical and lateral pressure during the obturation procedure. It just barely allows the use of a GP cone tapered according to the final shape of the canal, working as a key-andlock system. Without pressure, it is difficult for the filling materials to enter the isthmus regions, therefore, there was a possibility of more and larger voids forming in the isthmus in the $\mathrm{SC}$ technique group. This may be the main reason why more obvious voids can be observed under the operating microscope compared to the CWC and LC groups. In this study, the middle of the isthmus, which was $3 \mathrm{~mm}$ away from the apex,were horizontally crossed to observe voids. Through the microscope observation, it is obviously to find that among three obturation techniques, the middle part of the isthmus was mostly filled with root canal sealer even in CWC group, which indicated that the filling technique based on sealer was worthy of attention.

However, other authors reached different conclusions in their studies compared with this research. Iglecias et al. [15]measured the volume of voids in the mesial root of the human mandibular molar in the apical onethird showing Vertucci Type II configuration canals, and found no significant difference between the SC and CWC groups in the apical area. In addition, a similar study conducted by Keles et al. [18] used micro-CT to measure the volume of voids in the band-shaped isthmuses in the mesial root of human mandibular first molars filled with $\mathrm{AH}$ plus sealer, and found similarities between the SC and CWC groups. Somma et al. [26] assessed straight canals, and they found no differences in root canal obturation or void distribution when comparing these two techniques. Voids and gaps within root canal fillings can be influenced by several factors [18], such as the experience of the clinician, the root canal mechanical 
preparation technique, differences in irrigation methods, the selected filling technique, the physical properties of the selected sealer, the focused anatomy configuration of the root canal system, the use of different scanning devices, diverse calculation softwares and so on. We speculated that these factors may be reasons for the differences between other studies and the outcomes of this research.

In addition, it has been reported that the SC technique provides inadequate obturation in oval root canals [7, 27, 28]. A micro-CT analysis of band-shaped isthmuses revealed that both the roof and floor of this anatomy exhibited a long, oval cross-sectional shape [10], thus lacking a full adaptation to the oval canal walls would complicate the obturation of isthmuses using the SC technique. The CWC technique has been reported to provide a more homogenous filling with significantly fewer voids when compared to either the SC or the LC technique $[7,18,19,21,26,28]$. Homogeneous thermoplasticized GP with a certain liquidity, suitable vertical pressure, and tightly condensed backfilling make it more effective in filling complex root canal system [26-29]. In the present study, significant differences in the voids and more complete filling quality were found in the bandshaped isthmus region when comparing CWC with the cold-filling techniques. The better sealing,the better outcome for root canal therapy. Therefore, CWC technique may lead to a better prognosis for the tooth with bandshaped isthmus, which needed additional clinical studies to investigate.

However, Li et al. [30]demonstrated that the obturation quality, long-term outcome, and postoperative pain prevalence were similar between warm GP and cold LC obturation through a systematic review and meta-analysis. In addition, a retrospective analysis [31] reported that the overall success rate of nonsurgical endodontic treatment using EndoSequence Bioceramic Sealer and a single cone obturation technique was $90.9 \%$, which was higher than the reported success rate of initial root canal treatment $(89.1 \%)$ and retreatment $(85.6 \%)$ in a study with a large sample size [32]. Moreover, in a recent non-randomized clinical trial [33],the author used CBCT images and PA radiographs as loose criteria to compare the success rate of root canal treatments undertaken using a single cone technique and a calcium silicate sealer (BioRoot ${ }^{\text {TM }}$ RCS) with a warm vertical condensation technique and $\mathrm{AH}$ plus, and the results showed no difference between those two groups. These studies showed that although the CWC technique achieved a lower void percentage in isthmus areas in the present study, cold filling techniques still resulted in a good clinical outcome in some aspects, especially the SC technique. Thus, further research is needed.
There were several limitations to this study. The 3D-printed artificial teeth lacked the microscopic structures characteristic of the dentin of natural teeth such as dentinal tubules, therefore, the adhesion between the endodontic filling material and the root canal wall could not be completely reproduced. Another consideration was that heating could alter the properties of the sealer, thus impacting on results of obturation. In addition, the outcome of this study mainly based on one band-shaped isthmus type, but the different isthmus types such as V-shaped or other irregular configurations still remained important factors for the void distribution produced by root canal filling techniques.Therefore,further investigations on different isthmus types should be conducted to get more information on obturation efficacy with different techniques for treatment of these anatomically challenging variations.

\section{Conclusions}

Within the limitations of this in vitro study, our findings suggest that none of the root canal filling techniques produce void-free fillings in band-shaped isthmus obturation. The CWC yields superior obturation quality to that of SC and LC techniques in band-shaped isthmus area.

\section{Abbreviations \\ micro-CT: micro-computed tomography; SC: single-cone technique; CWC : continuous wave of condensation technique; LC: lateral condensation tech- nique; GP: gutta-percha; 3D: three dimensions; MB: mesial-buccal canal; ML: mesial-lingual canal; WL: working length.}

\section{Acknowledgements}

This research received funding from the National Natural Science Foundation of China [81570964].

\section{Authors' contributions}

Conceptualization/design/methodology; ZH Micro-CT Scanning/data acquisition; PZ and KY Formal analysis/ interpretation; QJ, FZ and ZH Original draft preparation, PZ, KY and QJ Review and editing in format and language; PZ and FZ Supervision; ZH. All authors have read and agreed to the published version of the manuscript.

\section{Funding}

This research received funding from the National Natural Science Foundation of China [81570964]. The funding bodies played no role in the design of the study and collection, analysis, and interpretation of data.

\section{Availability of data and materials}

All data involved in this study are available from corresponding author upon reasonable request.

\section{Declarations}

Ethics approval and consent to participate Not applicable.

Consent for publication

Not applicable. 


\section{Competing interests}

The authors declare that they have no competing interests.

\section{Author details}

'Department of Endodontics, Shanghai Ninth People's Hospital, Shanghai Jiao Tong University School of Medicine, College of Stomatology, Shanghai Jiao Tong University, Shanghai 200011, China. ${ }^{2}$ National Clinical Research Center for Oral Diseases, National Center for Stomatology; Shanghai Key Laboratory of Stomatology, Shanghai 200011, China.

\section{Received: 26 December 2020 Accepted: 22 April 2021}

\section{Published online: 01 May 2021}

\section{References}

1. Siqueira JF Jr, Rôças IN, Ricucci D, Hülsmann M. Causes and management of post-treatment apical periodontitis. Br Dent J. 2014;216(6):305-12.

2. Schilder H. Filling root canals in three dimensions. Dent Clin N Am. 1967;32:723-44

3. Alharmoodi R, Al-Salehi S. Assessment of the quality of endodontic re-treatment and changes in periapical status on a postgraduate endodontic clinic. J Dent. 2020;92:103261.

4. Vertucci FJ. Root canal anatomy of the human permanent teeth. Oral Surg Oral Med Oral Pathol. 1984;58(5):589-99.

5. Gillen BM, Looney SW, Gu LS, Loushine BA, Weller RN, Loushine RJ, et al. Impact of the quality of coronal restoration versus the quality of root canal fillings on success of root canal treatment: a systematic review and meta-analysis. J Endod. 2011;37(7):895-902.

6. Hommez GM, Coppens CR, De Moor RJ. Periapical health related to the quality of coronal restorations and root fillings. Int Endod J. 2002;35(8):680-9.

7. Alim BA, Garip Berker Y. Evaluation of different root canal filling techniques in severely curved canals by micro-computed tomography. Saudi Dent J. 2020:32(4):200-5.

8. Tavares PB, Bonte E, Boukpessi T, Siqueira JF Jr, Lasfargues JJ. Prevalence of apical periodontitis in root canal-treated teeth from an urban French population: influence of the quality of root canal fillings and coronal restorations. J Endod. 2009:35(6):810-3.

9. Ordinola-Zapata R, Bramante CM, Versiani MA, Moldauer BI, Topham G, Gutmann JL, et al. Comparative accuracy of the Clearing Technique, CBCT and Micro-CT methods in studying the mesial root canal configuration of mandibular first molars. Int Endod J. 2017;50(1):90-6.

10. Keleş A, Keskin C. A micro-computed tomographic study of band-shaped root canal isthmuses, having their floor in the apical third of mesial roots of mandibular first molars. Int Endod J. 2018:51(2):240-6.

11. von Arx T. Frequency and type of canal isthmuses in first molars detected by endoscopic inspection during periradicular surgery. Int Endod J. 2005;38(3):160-8.

12. Alves FR, Andrade-Junior CV, Marceliano-Alves MF, Pérez AR, Rôças IN, Versiani MA, et al. Adjunctive steps for disinfection of the mandibular molar root canal system: a correlative bacteriologic, micro-computed tomography, and cryopulverization approach. J Endod. 2016;42(11):1667-72.

13. Versiani MA, Alves FR, Andrade-Junior CV, Marceliano-Alves MF, Provenzano JC, Rôças IN, et al. Micro-CT evaluation of the efficacy of hard-tissue removal from the root canal and isthmus area by positive and negative pressure irrigation systems. Int Endod J. 2016:49(11):1079-87.

14. Celikten B, C FU, Tufenkci AIO, Misirli P. M, K OD, et al. Micro-CT assessment of the sealing ability of three root canal filling techniques. J Oral Sci. 2015;57(4):361-6.

15. Iglecias EF, Freire LG, de Miranda Candeiro GT, Dos Santos M, Antoniazzi $J H$, Gavini G. Presence of voids after continuous wave of condensation and single-cone obturation in mandibular molars: a micro-computed tomography analysis. J Endod. 2017;43(4):638-42.

16. Celikten B, Uzuntas CF, Orhan Al, Orhan K, Tufenkci P, Kursun S, et al. Evaluation of root canal sealer filling quality using a single-cone technique in oval shaped canals: an in vitro micro-CT study. Scanning. 2016;38(2):133-40.

17. Pedullà E, Abiad RS, Conte G, La Rosa GRM, Rapisarda E, Neelakantan P. Root fillings with a matched-taper single cone and two calcium silicatebased sealers: an analysis of voids using micro-computed tomography. Clin Oral Investig. 2020;24(12):4487-92.

18. Keleş A, Keskin C. Presence of voids after warm vertical compaction and single-cone obturation in band-shaped isthmuses using micro-computed tomography: a phantom study. Microsc Res Tech. 2020;83(4):370-4

19. Wolf M, Küpper K, Reimann S, Bourauel C, Frentzen M. 3D analyses of interface voids in root canals filled with different sealer materials in combination with warm gutta-percha technique. Clin Oral Investig. 2014;18(1):155-61.

20. Gandolfi MG, Parrilli AP, Fini M, Prati C, Dummer PM. 3D micro-CT analysis of the interface voids associated with Thermafil root fillings used with $\mathrm{AH}$ Plus or a flowable MTA sealer. Int Endod J. 2013;46(3):253-63.

21. Nguyen TA, Kim Y, Kim E, Shin SJ, Kim S. Comparison of the efficacy of different techniques for the removal of root canal filling material in artificial teeth: a micro-computed tomography study. J Clin Med. 2019;8(7):984.

22. Leoni GB, Versiani MA, Silva-Sousa YT, Bruniera JF, Pécora JD, Sousa-Neto MD. Ex vivo evaluation of four final irrigation protocols on the removal of hard-tissue debris from the mesial root canal system of mandibular first molars. Int Endod J. 2017;50(4):398-406.

23. Neelakantan P, Devaraj S, Jagannathan N. Histologic assessment of debridement of the root canal isthmus of mandibular molars by irrigant activation techniques ex vivo. J Endod. 2016;42(8):1268-72

24. Kim S, Jung H, Kim S, Shin SJ, Kim E. The influence of an isthmus on the outcomes of surgically treated molars: a retrospective study. J Endod. 2016;42(7):1029-34

25. Kim S, Kim S, Park JW, Jung IY, Shin SJ. Comparison of the percentage of voids in the canal filling of a calcium silicate-based sealer and gutta percha cones using two obturation techniques. Materials (Basel Switzerland) 2017;10(10):10-5.

26. Somma F, Cretella G, Carotenuto M, Pecci R, Bedini R, De Biasi M, et al. Quality of thermoplasticized and single point root fillings assessed by micro-computed tomography. Int Endod J. 2011;44(4):362-9.

27. Robberecht L, Colard T, Claisse-Crinquette A. Qualitative evaluation of two endodontic obturation techniques: tapered single-cone method versus warm vertical condensation and injection system: an in vitro study. J Oral Sci. 2012;54(1):99-104.

28. Hörsted-Bindslev P, Andersen MA, Jensen MF, Nilsson JH, Wenzel A. Quality of molar root canal fillings performed with the lateral compaction and the single-cone technique. J Endod. 2007;33(4):468-71.

29. Gok T, Capar ID, Akcay I, Keles A. Evaluation of different techniques for filling simulated C-shaped canals of 3-dimensional printed resin teeth. $J$ Endod. 2017:43(9):1559-64.

30. Peng L, Ye L, Tan H, Zhou X. Outcome of root canal obturation by warm gutta-percha versus cold lateral condensation: a meta-analysis. J Endod. 2007;33(2):106-9

31. Chybowski EA, Glickman GN, Patel Y, Fleury A, Solomon E, He J. Clinical outcome of non-surgical root canal treatment using a single-cone technique with endosequence bioceramic sealer: a retrospective analysis. J Endod. 2018;44(6):941-5

32. Ng YL, Mann V, Gulabivala K. A prospective study of the factors affecting outcomes of non-surgical root canal treatment: part 2: tooth survival. Int Endod J. 2011;44(7):610-25.

33. Zavattini A, Knight A, Foschi F, Mannocci F. Outcome of root canal treatments using a new calcium silicate root canal sealer: a non-randomized clinical trial. J Clin Med. 2020;9(3):782-91.

\section{Publisher's note}

Springer Nature remains neutral with regard to jurisdictional claims in published maps and institutional affiliations. 